\title{
Necropsias de neonatos, fator da melhoria da qualidade nas UTIs neonatais
}

\author{
Neonatal necropsies as a factor in quality improvement of the neonatal ICUs
}

Mônica Serapião ${ }^{1}$; Maria Jose Serapião²; Carlos Jose Serapião

\section{unitermos \\ UTIs neonatais \\ Necropsia pediátrica \\ Controle de qualidade}

\section{resumo}

Neste estudo foram estudados 552 casos de necropsia de neomortos provenientes de unidades de terapia intensiva neonatais (UTINs). Desses, 265 apresentaram algum tipo de enfermidade ou lesão não esperada conseqüentes (direta ou indiretamente) a seu manuseio diagnóstico e/ou terapêutico. Os casos foram agrupados por tipo de enfermidade, em seus respectivos órgãos ou sistemas, que ressaltou a prevalência de lesões pulmonares, como membrana hialina, enfisema intersticial, displasia broncopulmonar e alterações graves em nível de sistema nervoso central (SNC), sinalizando o prognóstico quanto à qualidade de vida. Como parte das medidas terapêuticas, devem ser analisados os acessos arteriais e venosos dos vasos umbilicais, com suas complicações, e o acesso venoso profundo, propriamente dito, para nutrição parenteral total, com graves complicações fatais para o lado do coração, como endocardite fúngica e tamponamento cardíaco por "Intralipid". Foram discutidas as resultantes multissistêmicas dos quadros de hipotensão e choque: enterocolite necrotizante e necroses corticomedular, renal, hepática e miocárdica. Este trabalho ressalta o valor da necropsia na melhoria da qualidade das UTINs, bem como apresenta várias situações em que o diagnostico só foi conhecido devido à necropsia ou, então, o resultado modificou, de certa forma, a abordagem terapêutica futura. A consulta e a análise da literatura demonstram a virtual inexistência de metodologia adequada para desenvolver e estabelecer um comportamento que propicie o exercício sistemático de aferição do desempenho organizacional, e que reduza sensivelmente as possibilidades de efeitos indesejáveis relacionados com rotinas e procedimentos operacionais nesse campo da prática assistencial. As principais causas de insucesso parecem ser creditadas à tecnologia de máquinas e substâncias, cuja adequação à biologia dos organismos em desenvolvimento não é plenamente conhecida no que se refere à influência exercida por um grande número de variáveis fisiopatológicas atuantes no procedimento terapêutico.

\section{abstract}

The present study emphasizes the necropsy value in the development of the neonatal ICU. We present many situations where the diagnostic was possible solely because of the necropsy, as well, many diagnosis were changed based on the necropsies results. The literature compilation shows no evidence of a systematic procedure concerning the mitigation of the problems related to the avaliable routines in this matter. The lack of a more scientific investigation related to the neonatal deaths is a enormous barrier to the improvement of those organizations (ITU's). Apparently, these failure is connected to machines and products thecnology not well know in terms of their suitability concerning under biological development organisms. Also, the necropsies appears to be a useful tool when the death results, directly or indirectly, from therapeutical process. The conception of a quality development process strategy represents a major issue, even more when you face new political decisions in health field, including cost reduction and higher complexity. Also, we need to pay special attention to science and research ethical principles. key words

Neonatal ICUS

Pediatric necropsy

Quality improvement 


\section{Introdução}

A implementação de estratégias para melhoria da qualidade dos processos e serviços realizados em unidades de terapia intensiva neonatais (UTINs), visando alcançar gestão social dos recursos públicos e privados na área da saúde mais eficiente, atualmente se encontra no vértice da pirâmide de prioridades em todo o mundo.

Portanto, justifica-se o investimento de esforços de tantos quanto estejam envolvidos no estudo dos problemas dessa difícil área, em busca de soluções práticas e imediatas que possibilitem transformações capazes de otimizar o desempenho de instituições e profissionais, protagonistas dessas complexas ações assistenciais, a fim de se obter a definição de um novo paradigma para a saúde que não incorpore como ingrediente básico o aumento de recursos naquela área, o que já está provado é a mais difícil das soluções a ser praticada em nossos dias.

Por outro lado, o conhecimento das lesões que acompanham, sob a forma de efeitos adversos, muitas ações terapêuticas praticadas nas UTINs oferece um sinal de alerta para a existência de respostas indesejáveis, devendo ser considerado por ocasião do monitoramento e avaliação do neonato enfermo e da terapêutica que lhe é instituída.

Assim, o patologista assume destacada importância visto que, com freqüência, é a primeira pessoa a chamar atenção para o efeito adverso de nova aquisição em meio a esse arsenal terapêutico.

Só com alto índice de necropsias realizadas através de protocolos padronizados é possível identificar e reunir número suficiente de eventos de uma determinada lesão e, desse modo, considerá-la relacionada com uma ou outra manobra terapêutica.

Em geral, no terreno da terapia intensiva neonatal, as lesões produzidas por um procedimento, substância ou manobra específica, podem ser agrupadas em três grandes categorias:

- a primeira categoria reúne lesões que são diretamente relacionadas com o processo da qual elas resultaram, ou pode representar uma inevitável conseqüência desse procedimento. Por exemplo: erosões na traquéia como resultado de intubação traqueal, e trombos em redor da extremidade de cateteres intravasculares ou intracardíacos;

- a segunda inclui lesões cuja relação não é diretamente relacionada com o procedimento que as causaram e, nesse caso, se apresentam como um efeito indesejável ou inesperado. Um bom exemplo é constituído pela colestase que se associa à nutrição parenteral.
- a terceira categoria, que pouco a pouco vem ampliando, envolve lesões conseqüentes à sobrevida prolongada oferecida aos pequenos pacientes.

Muitas dessas lesões surgem como evolução esperada de um evento precursor que vai se instalando gradativamente. Como exemplo, pode ser citada a estenose ou atresia do intestino, como resultado de um insulto isquêmico no recém-nato, ou os cistos da porencefalia, os quais resultam do evento biológico da recuperação ou cura do processo isquêmico cerebral, que desempenha papel crucial no resultado extremo do processo.

O estudo dessas lesões, chamando atenção para as mais freqüentes e as distribuindo por órgãos ou sistemas, é a forma mais usual da literatura, pois apresenta a conveniência de também agrupá-las pelo mecanismo patogenético envolvido como, por exemplo, as que resultam da respiração assistida, do uso de vias parenterais, e da exposição ao risco de infecções por serem de procedimentos invasivos etc.

A possibilidade de que uma lesão possa se originar de procedimento terapêutico tem aumentado o nível de litígio e de ansiedade entre leigos e profissionais de saúde. Entretanto, o reconhecimento da existência dessas condições indesejáveis introduziu um recomendável sentido de cautela, associado a maior rigor e alerta no monitoramento e avaliação de recém-natos enfermos, assim como nos procedimentos terapêuticos instituídos. Todos esses fatores contribuem para que se cuide melhor do paciente crítico $^{(25)}$.

Para uma atuação correta, o patologista necessita ser informado de todos os procedimentos e terapêuticas utilizados no atendimento ao recém-nato. É igualmente importante realizar necropsia completa, incluindo, quando aplicáveis, cariótipo e investigações bioquímicas, microbiológicas e radiológicas.

É importante recomendar que todos os tubos e cateteres sejam deixados em suas posições originais, para que essas informações possam ser compartilhadas com o patologista. Essa simples precaução pode ser significativa para evitar o risco de se perder uma valiosa contribuição para o esclarecimento do efeito adverso de uma manobra terapêutica.

Existem múltiplos fatores capazes de gerar confusão quando são discutidos os efeitos e as complicações da terapêutica nos recém-natos. Um exemplo clássico é citado por Cross $^{(10)}$ referente a seu registro sobre as muitas restrições impostas à oxigenoterapia na Inglaterra, nas décadas de 50 
e 60, que resultou numa significativa redução do número de casos de fibroplasia retrolenticular (retinopatia do prematuro). Todavia, isso foi obtido à custa de um aumento nas mortes nas primeiras 24 horas de vida.

Numa visão ampla, grande parte das lesões relacionadas com os cuidados intensivos neonatais pode ser prevista, e quase sempre há uma complexa inter-relação com múltiplas possíveis causas. Nem todas as alterações se devem às imediatas conseqüências da terapêutica instituída. Por outro lado, muitas lesões distintas, em diferentes órgãos, podem coexistir em um mesmo paciente no qual uma alteração pode induzir o desenvolvimento de mais uma lesão em outro órgão. Assim, displasia broncopulmonar, cicatrizes de fibrose miocárdica e lesões císticas nos hemisférios cerebrais podem coexistir, e realmente coexistem, no mesmo paciente.

A patogenia de muitas dessas alterações pode estar interrelacionada. Lesão em um órgão ou sistema pode modificar ou facilitar alterações em outros órgãos, ou mesmo um único procedimento pode provocar múltiplos efeitos ${ }^{(19)}$.

Para o patologista que não esteja afeito a esse tipo de material resultante da prática perinatal, podem surgir dificuldades na interpretação dos achados morfológicos. Portanto, não se pode prescindir de informes clínicos adequados que previnam erros no julgamento diagnóstico ${ }^{(45)}$.

Entre as práticas terapêuticas correntes pré e pós-natais, que se relacionam com o risco de surgimento de efeitos indesejáveis, podem ser citadas como as mais freqüentes:

- terapêuticas materna e fetal por drogas $(4,29,44)$;

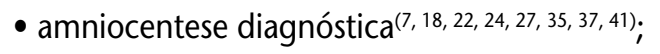

- cordocentese umbilical ${ }^{(6,8)}$;

- transfusão intra-uterina(4);

- biópsia de vilosidade coriônica(18,43);

- prevenção de parto prematuro(21, 22, 28);

- intubação traqueal ${ }^{(12,38)}$;

- cateterismos arterial e venoso(1, 3, 12, 20, 40, 42);

- nutrição parenteral(1, 2,32-34,36,39).

\section{Objetivos}

Este trabalho procurou ampliar o conhecimento das causas e mecanismos de morbimortalidade como um dos mais significativos parâmetros da assistência médica em uma unidade de terapia intensiva neonatal (UTIN), por meio da análise dos insucessos, além dos seus componentes causais, realizando um estudo estruturado importante para a sistematização das propostas de ações preventivas.

O principal objetivo foi oferecer informações analisadas, sob o ponto de vista anatomopatológico, que permitissem estabelecer uma estratégia de melhoria na qualidade das práticas assistenciais nas UTINs e que, ao mesmo tempo, contribuíssem para um desempenho melhor de seus profissionais e para o aperfeiçoamento do processo.

Foi utilizado como instrumento aquele que permitiu:

- identificar as principais causas de mortes neonatais relacionadas com os procedimentos diagnósticos e/ou terapêuticos no grupo estudado;

- sistematizar os procedimentos ou grupos de procedimentos que propiciaram maior risco de surgimento de respostas indesejáveis;

- reafirmar que o patologista pode oferecer por meio da necropsia informes importantes para essa melhoria do processo.

\section{Material e métodos}

Em um universo de 1.700 necropsias de pacientes falecidos no período perinatal, necropsiados no LUPA Laboratórios Unidos de Patologia, no Rio de Janeiro, num período de 20 anos (1976 a 1996), foram isolados 552 neomortos provenientes de nove UTINs dessa cidade, entre os quais foram identificadas e selecionadas 265 necropsias cujos óbitos apresentaram relação com um evento considerado indesejado como causa de morte, básica ou associada.

Realizamos todas as necropsias utilizando idêntica metodologia, a preconizada e consagrada pela prática anatomopatológica, e posteriormente essas necropsias foram submetidas a estudos macro e microscópico completos, bem como a documentação fotográfica/radiológica (post mortem) dos achados significativos. Os preparados histológicos foram inicialmente corados por hematoxilina-eosina, a partir dos quais foram selecionados para técnicas especiais que incluíram: Masson, retículo de Gomori, Giemsa, Gram, Grocott, Wade, orceína, Perls com suas finalidades específicas, visando complementar a interpretação das alterações morfológicas observadas.

Todos os achados das necropsias macro e microscópicos foram relacionados e distribuídos de acordo com a localização e/ou etiopatogenia, inclusive os que não estiveram diretamente relacionadas com a causa da morte. 
Todos os pacientes provenientes de UTIs neonatais apresentavam cultura de superfície cutânea, swab nasal e de orofaringe, com cultura e hemocultura em várias etapas do período de internação. As patologias infecciosas identificadas nas necropsias estão sumariadas na Tabela 5.

\section{Resultados}

Os resultados referentes aos diagnósticos obtidos pelas necropsias, bem como as alterações observadas de modo concomitante nos vários pacientes, criaram a necessidade de agrupá-los dentro de sistemas anatômicos (Tabelas 1 a 4) e mecanismos lesionais básicos (Tabelas 5 a 8), a fim de possibilitar melhor avaliação. Dessa forma, eles estão expressos em suas freqüências absolutas e percentuais, em relação com as 265 necropsias, nas Tabelas 1 a 8.

\section{Tabela 1 Patologia do sistema respiratório}

\begin{tabular}{lcc}
\hline Diagnóstico & $n$ & $\%$ \\
- Enfisema intersticial pulmonar & 84 & 31 \\
- Membrana hialina & 80 & 30 \\
- Lesão de laringe e traquéia & 57 & 21 \\
- Displasia broncopulmonar & 45 & 17 \\
- Hemorragia pulmonar & 32 & 12 \\
- Pneumonias & 30 & 11 \\
- Pneumotórax & 28 & 10,5 \\
- Pneumonia intra-uterina & 8 & 3 \\
- Hemotórax & 5 & 1,9 \\
- Embolia gasosa & 2 & 0,8 \\
- Enfisema pulmonar intersticial & 1 & - \\
- Persistente & & \\
- Perfuração de brônquio & 1 & - \\
\hline
\end{tabular}

\section{Tabela 2 Patologia do sistema nervoso central}

\begin{tabular}{lcc}
\hline Diagnóstico & $n$ & $\%$ \\
- Hemorragias peri e intraventricular & 66 & 25 \\
- Leucomalacia periventricular & 52 & 19,5 \\
- Necrose neuronal seletiva & 28 & 10,5 \\
- Meningite neonatal & 16 & 6 \\
- Hemorragias epi e subdural & 9 & 3,3 \\
- Hemorragia subaracnóide & 9 & 3,3 \\
- Hemorragia cerebelar maciça & 8 & 3 \\
- Kernicterus & 7 & 2,6 \\
- Injúria parassagital cerebral & 3 & 1,1 \\
- Necrose ponto-subicular & 3 & 1,1 \\
- Hemorragia intraventricular & 2 & 0,75 \\
- (plexo coróide) & & \\
- Trombose de seios durais & 2 & 0,75 \\
\hline
\end{tabular}

Tabela 3

\section{Patologia do sistema cardiovascular}

\begin{tabular}{lcc}
\hline Diagnóstico & $n$ & $\%$ \\
- Cateterismo de artérias umbilicais* $^{*}$ & 69 & 26 \\
- Cateterismo de veia umbilical & 43 & 16,2 \\
- Cateterismo venoso profundo* & 18 & 6,8 \\
- Reação granulomatosa em parede & 12 & 4,5 \\
$\quad$ vascular & & \\
- Tamponamento cardíaco & 2 & 0,75 \\
\hline
\end{tabular}

* Número de casos com lesões resultantes do respectivo cateterismo.

\begin{tabular}{lcc}
\hline Tabela 4 & Patologia cutânea \\
\hline Diagnóstico & $n$ & $\%$ \\
\hline - Complicações cutâneas & 29 & 10,9 \\
\hline
\end{tabular}

\section{Tabela 5 Patologia infecciosa}

\begin{tabular}{lcc}
\hline Diagnósticos & $n$ & $\%$ \\
\hline - Infecções fúngicas & 30 & 11,3 \\
- Pneumonias & 30 & 11,3 \\
- Meningite neonatal & 16 & 6 \\
- Sépsis por gram-negativos & 15 & 5,6 \\
- Pneumonias intra-uterinas & 8 & 3 \\
- Citomegalovírus adquirido & 2 & 0,75 \\
- Adenovírus & 2 & 0,75 \\
- Herpes generalizado & 1 & - \\
- Vírus sincicial respiratório & 1 & - \\
\hline
\end{tabular}

\section{Patologia associada a quadro}

Tabela 6 hipóxico/isquêmico

\begin{tabular}{lcc}
\hline Diagnóstico & $n$ & $\%$ \\
- Leucomalacia periventricular & 52 & 19,5 \\
- Necrose miocárdica & 31 & 11,7 \\
- Enterocolite necrotizante & 24 & 9 \\
- Necrose cortical/corticomedular & 19 & 7,1 \\
$\quad$ renal & & \\
- Necrose hepática & 11 & 4,1 \\
- Injúria parassagital cerebral & 3 & 1,1 \\
- Perfuração gástrica & 2 & 0,75 \\
\hline
\end{tabular}




\begin{tabular}{lcc} 
Tabela 7 & Patologia hemorrágica & \\
\hline Diagnóstico & $n$ & $\%$ \\
- Hemorragia pulmonar & 32 & 12 \\
- Hemoperitônio & 13 & 4,9 \\
- Hemotórax & 5 & 1,9 \\
- Hemopericárdio & 4 & 1,5 \\
- Hematoma de adrenal & 3 & 1,1 \\
- Hemomediastino & 1 & - \\
\hline
\end{tabular}

\begin{tabular}{lccc} 
Tabela 8 & Patologia da nutrição parenteral \\
\hline Diagnóstico & $n$ & $\%$ \\
\hline - Complicações da nutrição parenteral & 27 & 10,2 \\
\hline
\end{tabular}

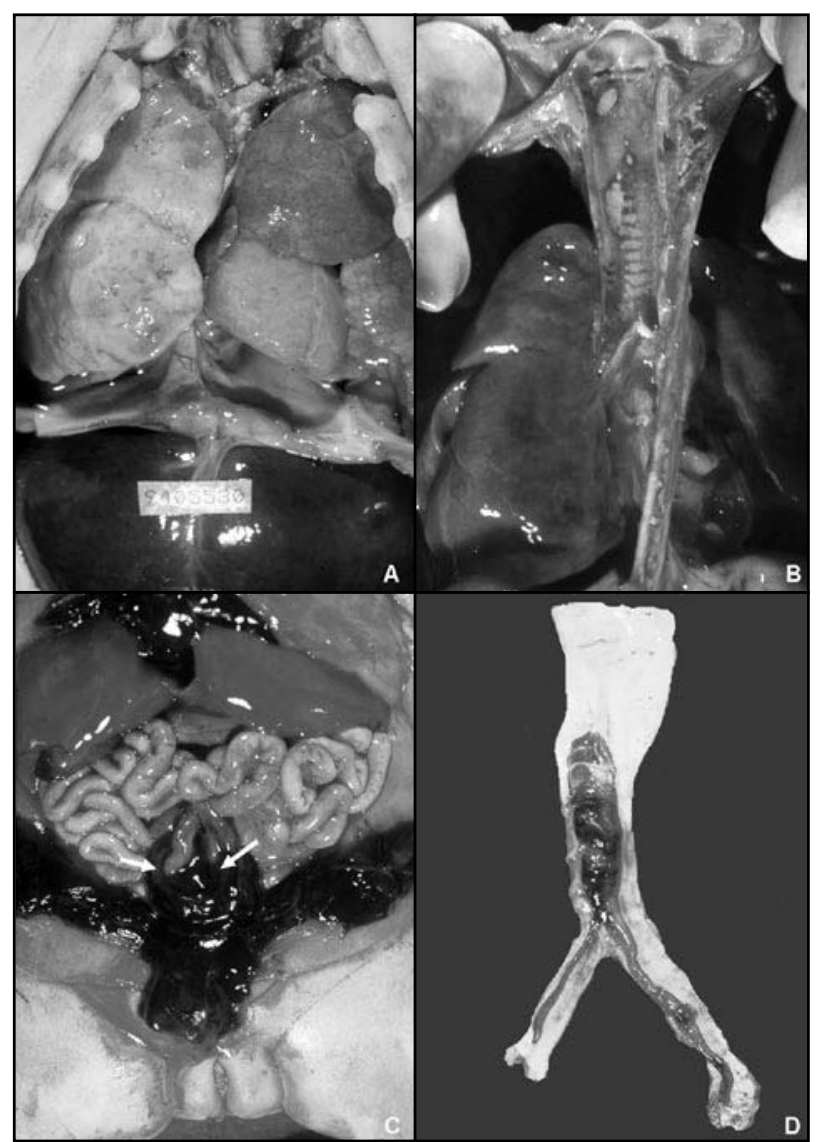

Figura 1 - A. Enfisema intersticial: pulmões preenchendo completamente as cavidades pleurais. B. Laringe e traquéia com áreas de necrose: intubação orotraqueal, sépsis por Klebsiela. C. Neomorto a termo: hemorragia volumosa em torno de ambas artérias umbilicais. $D$. Trombo organizado em aorta abdominal se estendendo para ambas ilíacas primitivas
A seguir são listados alguns dos diagnósticos que não puderam ser suspeitados em vida:

- trombose de artérias umbilicais com hemorragia em torno da adventícia ou retroperitônio;

- trombose de veia umbilical com infecção introduzida pelo cateter;

- endocardite fúngica de tricúspide;

- tamponamento cardíaco por Intralipid;

- perfuração gástrica;

- kernicterus;

- alterações microscópicas hepáticas e pancreáticas ligadas à nutrição parenteral;

- lesões do sistema nervoso central (SNC) por mecanismos hipóxico-isquêmico e hemorrágico somente puderam ser adequadamente sistematizadas com o exame do encéfalo (Tabela 2).

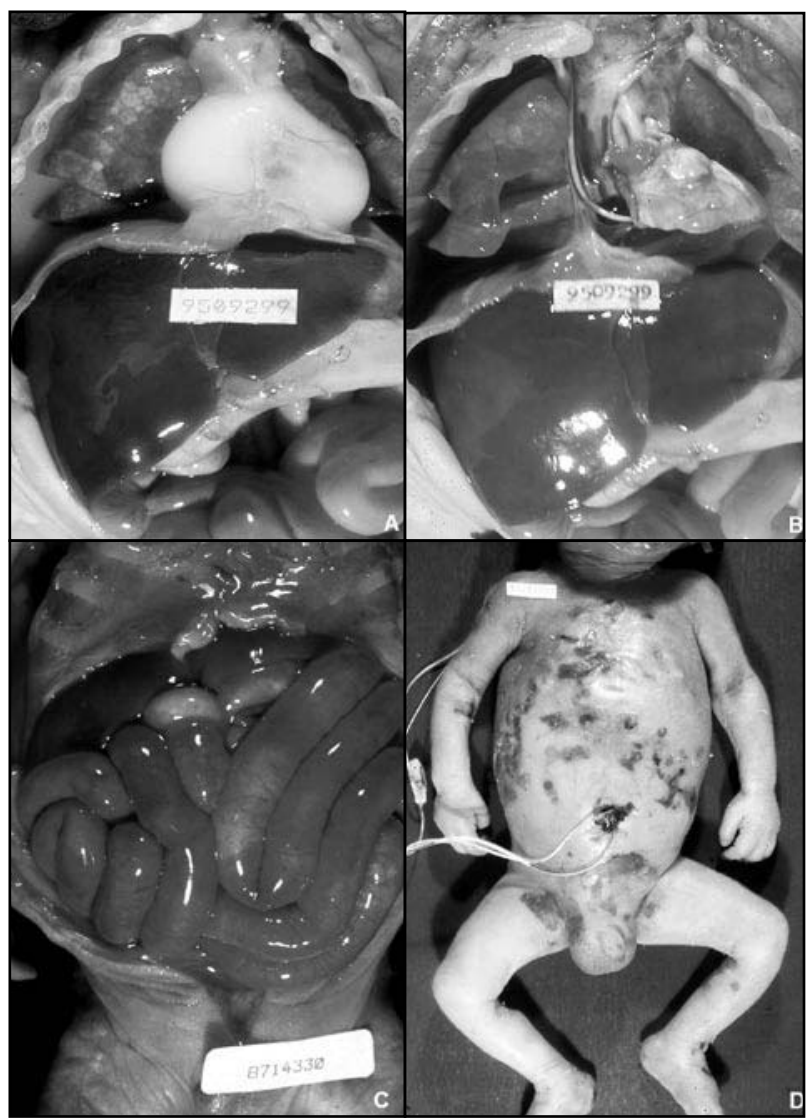

Figura 2 - A. Tamponamento cardíaco por Intralipid. Observar saco pericárdico distendido contendo líquido de aspecto leitoso, presente também na cavidade pleural direita. B. Cava superior e aurícula direita abertas com cateter. C. Cavidade abdominal aberta, alças intestinais distendidas e de tonalidade vinhosa. D. Pele do abdome, tórax e perineo exibindo lesões ulcerocrostosas disseminadas por acessos venosos, transdutores e drenagens. Artéria e veia umbilical cateterizadas 


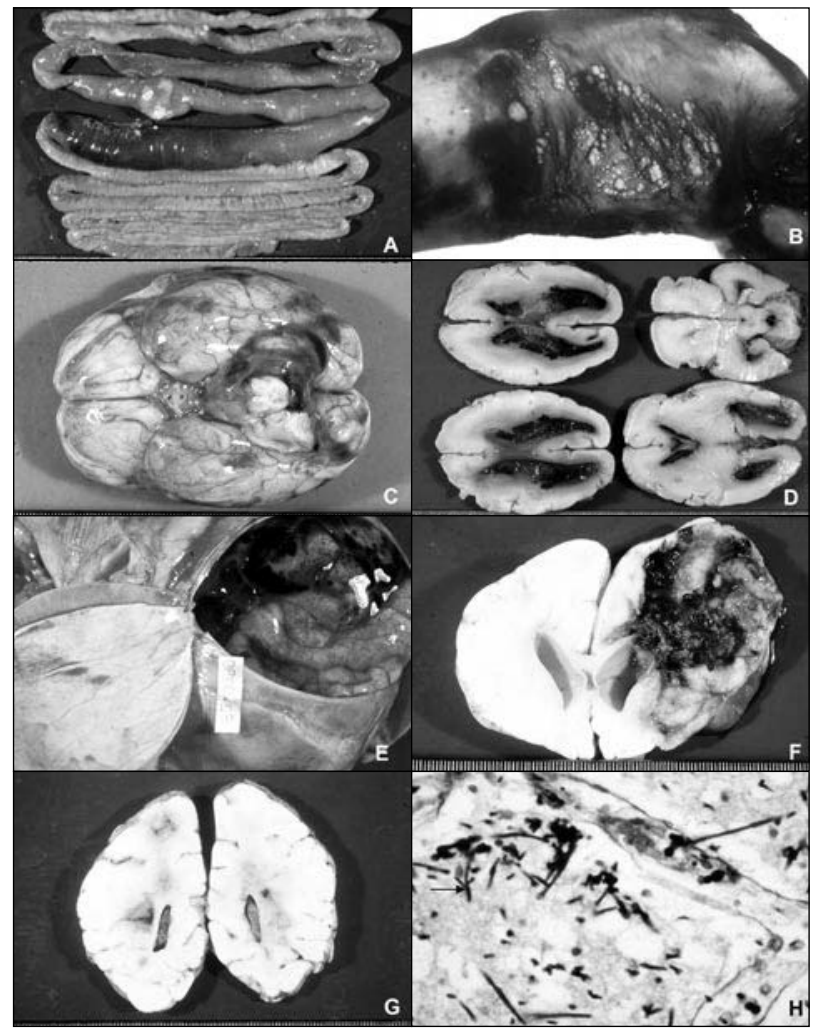

Figura 3 - A. Intestino delgado e grosso em toda sua extensão, mostrando enterocolite necrotizante expressa em vários segmentos. B. Pneumatose do cólon visto por transiluminação. Observar bolhas de ar na parede intestinal. C. Neomorto pré-termo. Encéfalo com exsudato purulento na base. Meningite neonatal por Enterobacter rafnia. D. Do mesmo caso: cortes horizontais do cérebro mostram hemorragias peri e intraventricular grau III. E. Crânio: janela parietal aberta mostra extensa necrose hemorrágica do parênquima nervoso. F. Do mesmo caso: corte frontal na altura do pólo frontal mostra destruição do parênquima. $\mathrm{G}$. Corte frontal de hemisférios cerebrais leucomalacia periventricular. $\mathrm{H}$. Grocott 400x - mesmo caso: encefalite por Candida sp. (septicemia fúngica)

\section{Comentários}

Em geral, os recém-nascidos internados em UTINs se enquadram como prematuros, recém-nascidos a termo que sofreram asfixia de moderada a severa, e portadores de malformações congênitas que necessitam de terapia intensiva ou algum tipo de correção cirúrgica.

\section{Sistema respiratório (Tabela 1)}

Em relação aos prematuros, há que se destacar a grande freqüência de enfermidades respiratórias, como a doença de membrana hialina, quando é necessária oxigenação suplementar com ventilação assistida e intubação orotraqueal.

A utilização de pressões de oxigênio em níveis adequados para um recém-nascido, tendo em vista a sua capacidade torácica e dependendo da doença pulmonar básica, poderá produzir enfisema intersticial pulmonar, agravando ainda mais o quadro respiratório, que pode se complicar e levar a pneumotórax e até mesmo pneumopericárdio (Figura 1A).

Em casos extremos pode ocorrer embolia gasosa, como em duas situações observadas nesta série estudada. Se o ar progride para o subcutâneo, advém enfisema subcutâneo que, ao alcançar o abdome, ocasiona pneumoperitônio, configurando desse modo todo o espectro do quadro de barotrauma.

Em nossa amostra estudada houve grande prevalência dessas complicações, tanto em prematuros como em recémnascidos a termo, com grau importante de asfixia periparto e aspiração de líquido amniótico e/ou mecônio. Nestes últimos, há dificuldade de oxigenação produzida pela aspiração, associada a grande resistência da circulação pulmonar pelo não-relaxamento da parede das arteríolas musculares, que deveria ocorrer no momento do parto ${ }^{(14,25,30)}$.

Desse modo, a circulação do tipo fetal permanece, condicionando o shunt direito/esquerdo, que determina aumento crescente nos níveis pressionais da ventilação assistida, no sentido de se conseguir uma razoável oxigenação do paciente (pressão parcial de oxigênio [PO2]). Rompem-se, então, os septos alveolares com conseqüente enfisema intersticial, acumulando ar ao longo dos septos interlobulares e por baixo da pleura.

A pressão não necessita ser tão elevada para que haja lesão, pois com graus variáveis de obstrução brônquica/ bronquiolar o ar não se distribui de maneira homogênea. Como complicação também encontramos hemorragias pulmonares, que podem ser focal, multifocal ou comprometer um segmento ou um lobo pulmonar.

\section{Alterações na traquéia e brônquios}

Mesmo em mãos habilidosas, há a possibilidade de se produzir trauma na laringe, traquéia ou brônquio durante a introdução do tubo.

A presença do tubo orotraqueal lesando a delicada mucosa da laringe e da traquéia abre uma porta de entrada para infecções. Em nosso material foram mais prevalentes as infecções por germes gram-negativos, como Pseudomonas e Klebsiella, e as infecções fúngicas, principalmente por Candida sp. (Figura 1B). Essas infecções podem ser localizadas ou generalizadas e com gravidade variável, chegando em casos extremos a provocar a morte do paciente.

A mucosa sofre necrose com exsudação leucocitária e grande colonização pelo germe invasivo, comprometendo 
o córion mucoso, as glândulas e, em alguns casos, expondo e destruindo parcialmente a cartilagem da laringe ou do anel traqueal.

Outra condição observada é a traqueobronquite necrotizante, que compromete a porção mais inferior da traquéia, podendo se estender aos brônquios fontes ${ }^{(15)}$. É uma lesão distal ao tubo orotraqueal, portanto não pode ser atribuída a trauma mecânico. Há ainda a formação de uma pseudomembrana aderente à mucosa. Atribui-se como fator etiológico da lesão, uma injuria térmica ou tóxica pelos componentes do tubo e pelo ressecamento ${ }^{(5,26)}$.

\section{Displasia broncopulmonar}

A displasia broncopulmonar é uma entidade que surgiu após o aparecimento das UTINs. Resulta do desenvolvimento anormal do pulmão de recém-nascidos com doença da membrana hialina, sob o efeito da hiperoxigenação e do barotrauma, em um período de tempo prolongado e variável. Anteriormente, ela não ocorria porque os recém-nascidos com membrana hialina grave terminavam em óbito precoce, não havendo tempo da lesão se estabelecer.

Os pacientes portadores de membrana hialina foram, entre outros, os grandes beneficiados pela existência das UTINs. Essa enfermidade é mais prevalente em recémnascidos prematuros, e reflete uma imaturidade do parênquima pulmonar, com baixa produção de surfactante pelos pneumócitos II.

Essa substância recobre os alvéolos, diminuindo a tensão superficial que permite sua expansão. A displasia broncopulmonar é uma seqüela da insuficiência de surfactante às vezes inevitável, mas que varia de acordo com a gravidade do processo inicial. Hoje se tornou mais controlável devido ao surgimento no arsenal terapêutico de substâncias surfactantes inalatórias ${ }^{(9,10)}$.

Em nossos casos, o aspecto histológico dos pulmões dos pacientes tratados com surfactante que foram a óbito não mostrou diferenças significativas.

\section{Trauma}

Lesões mecânicas traumáticas foram raras e encontradas em três casos: uma perfuração de brônquio na vigência de broncoscopia, acarretando enfisema de mediastino e enfisema subcutâneo cervical; e dois casos de perfuração de esôfago com mediastinite posterior.

\section{Sistema cardiovascular (Tabela 3)}

\section{- Acesso arterial}

Em qualquer situação de risco é imperioso que haja um acesso vascular. Nos recém-nascidos é comum a utilização de artérias umbilicais e veia umbilical, já que são vasos razoavelmente calibrosos e de mais fácil acesso.

As artérias umbilicais são utilizadas principalmente para monitorar os gases sangüíneos arteriais. Determinadas pelas tentativas de cateterização, foram produzidos vários casos de falso trajeto, com perfuração da respectiva artéria e hemorragia, que, em alguns casos, se restringiu à adventícia ou se estendeu aos tecidos periumbilicais e perivesicais, formando hematomas volumosos na pelve e retroperitônio, o que levou à morte por choque hipovolêmico (Figura 1C) ${ }^{(3,42)}$. Esses casos mais graves ocorrem quando a primeira tentativa de cateterização se realiza após algumas horas do parto, pelo fato de que o vaso já iniciou sua contração fisiológica.

Nos primeiros casos em que houve tal complicação, o diagnóstico não foi suspeitado em vida e somente pela necropsia ficou estabelecida a causa mortis. Existem poucos casos relatados na literatura ${ }^{(40)}$.

Com base nesses achados, os neonatologistas passaram a se prevenir para um possível acidente dessa natureza, e tomaram como precaução não tentar fazer progredir o cateter quando houvesse um mínimo de impedimento. Mesmo em casos nos quais havia a remota possibilidade de ruptura do vaso, recorreu-se à ligadura da artéria umbilical, baseando-se em dados clínicos, como palidez, hipotensão e taquicardia.

A localização da extremidade do cateter na aorta deve ser na altura da $L 2$ ( $\pm 1,5 \mathrm{~cm}$ acima da bifurcação da aorta) ou na altura do diafragma, para proteger a emergência das artérias renais e mesentéricas. A trombose de artérias umbilicais, ilíacas primitivas e aorta foi freqüente, com suas respectivas conseqüências (Figura 1D).

Houve casos de trombose aórtica com obstrução da emergência das artérias renais e conseqüente necrose corticomedular renal. Também para o membro inferior do lado cateterizado, foram observadas complicações, com palidez relacionada com o vasoespasmo, e necrose de pododáctilos resultantes de fenômenos trombembólicos.

Em nossa experiência, os cateteres arteriais não representaram porta de entrada para a infecção, o que está de acordo com a literatura ${ }^{(12)}$. 


\section{- Acesso venoso}

A veia umbilical é cateterizada para infusões de líquidos e medicamentos. $\mathrm{O}$ trajeto ideal do cateter é a veia umbilical, o ducto venoso de Aranzio e a veia cava inferior. Não raramente o cateter alcança a veia porta, se alojando em seus ramos principais à direita ou à esquerda, ou em algum ramo intra-hepático. $\mathrm{O}$ medicamento introduzido que deveria se diluir na circulação em geral, nesta posição se concentra no fígado, lesando o parênquima.

Trombose de veias umbilical e porta e/ou seus ramos intra-hepáticos foi freqüente. Também de gravidade considerável é a introdução de agentes infecciosos via cateter, provocando infecção na parede da veia porta e infecção sistêmica. Alguns casos de candidíase generalizada tiveram como porta de entrada o cateter da veia umbilical ${ }^{(1)}$.

Complicações graves também foram observadas nos casos de cateterismo venoso profundo, como, por exemplo, para nutrição parenteral total. Foram evidenciadas tromboses de jugular interna, cava superior, átrio direito, válvula tricúspide e ventrículo direito.

Encontramos cinco casos de endocardite fúngica da tricúspide com vegetações volumosas, sendo um deles também com vegetação na câmara de saída do ventrículo direito, provocando hipofluxo pulmonar. Em dois casos foi identificado o fungo Malassezia furfur nas vegetações, as quais estavam ligadas à infusão de Intralipid. Esse fungo requer altas concentrações de ácidos graxos para seu crescimento - 0 agente Malassezia provavelmente estava se multiplicando no próprio Intralipid ${ }^{(33,34)}$. Em nenhum desses casos a etiologia da endocardite foi elucidada em vida.

É de se ressaltar também, dada a sua raridade na literatura, dois casos de tamponamento cardíaco por Intralipid, devido à perfuração do ventrículo direito, com escape do líquido para dentro da cavidade pericárdica (Figura $2 \mathrm{~A}$

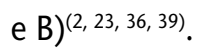

O material lipídico infundido, ao atingir a circulação pulmonar, pode se acumular na íntima, média e adventícia das artérias pulmonares, reduzindo a luz dos vasos e potencializando a hipertensão pulmonar. O lipídio é fagocitado pelos macrófagos que são observados no interior das cavidades alveolares, limitando seus mecanismos de defesa contra infecções.

Ainda como conseqüência de acessos vasculares, múltiplos pequenos corpos estranhos (fios de algodão, fragmentos de pêlo etc.) podem ter acesso à circulação e se alojar na parede de vasos pulmonares, provocando reação granulomatosa do tipo corpo estranho, em qualquer camada do vaso, na íntima, na qual são englobados pelo endotélio, na média, ou na adventícia. Favara e Moors ${ }^{(13)}$ encontraram maior prevalência em pacientes submetidos à cirurgia cardíaca.

De um modo geral, esse evento foi um achado microscópico e não conseguimos correlacionar com um quadro clínico específico. Encontramos 12 casos desse tipo de lesão, mas em nenhum deles a intensidade foi grande.

\section{Alterações hipóxico-isquêmicas (Tabela 6)}

\section{- Gastrintestinais, miocárdicas e renais}

Foram diagnosticados dois casos de perfuração gástrica que levaram a pneumoperitônio. Eram duas crianças asfixiadas (uma pré-termo e outra a termo) que utilizavam sondas nasogástricas. Surge então uma questão, a de se atribuir ao efeito propriamente dito da sonda na etiologia da perfuração, com todas as conseqüentes implicações que o fato acarreta.

Todavia, a seqüência mais lógica seria considerar uma importante injúria hipóxico-isquêmica no tubo gastrintestinal, com necrose da mucosa e, em graus variáveis, da parede, somada à distensão gástrica provocada pelas manobras de ventilação. Tal distensão torna a parede menos perfundida e delgada, propiciando sua ruptura. A sonda seria, no máximo, o agente de localização(11). Nenhum dos dois casos foi diagnosticado em vida; houve apenas o diagnóstico de pneumoperitônio.

O mecanismo hipóxico-isquêmico esplâncnico também é a base etiológica da enterocolite necrotizante, uma complicação temida principalmente nos recém-nascidos prematuros com dificuldade respiratória e nos a termo com asfixia severa (Figuras 2C; 3A e 3B).

Os eventos periparto que levam à asfixia, à prematuridade e a distress respiratório, tornando necessária a ventilação assistida, freqüentemente levam a quadros de choque, com hipotensão e sofrimento de múltiplos órgãos, notadamente SNC, coração, fígado e rins.

Deve-se discutir também o papel da indometacina nesses eventos isquêmicos. Essa droga é utilizada para fechar o canal arterial sem recorrer à cirurgia, e pode da mesma maneira afetar o leito vascular em outras localizações ${ }^{(12)}$.

A extensão da lesão miocárdica, representada pela necrose, será responsável pelo prognóstico imediato da criança, enquanto a extensão da lesão do SNC, o prog- 
nóstico de longo prazo, será caracterizada pelas seqüelas neurológicas.

Nos sobreviventes é comum encontrar graus variáveis de necroses de fibras miocárdicas, principalmente em músculos papilares em processo de cicatrização, com fibrose e mesmo calcificação. Nos casos mais graves essas são antecipadas pelas alterações no eletrocardiograma (ECG).

Ainda no espectro das alterações hipóxico-isquêmicas, vale referir as necroses corticomedular renal e hepática.

\section{Sistema nervoso central}

Em um percentual significativo de casos, o SNC é comprometido nos pacientes provenientes de UTINs (Tabela 2). São padrões lesionais ligados à prematuridade, como a hemorragia perintraventricular cerebral (Figura 3D); relacionadas com hipoxemia, como a necrose neuronal seletiva; lesões associadas à isquemia, como a leucomalacia periventricular (Figura 3 (E, F e G), a injuria cortical parassagital e a necrose ponto-subicular; a impregnação biliar de neurônios corticais e de núcleos profundos do encéfalo, como conseqüência de hiperbilirrubinemia, caracterizando o kernicterus (kern = núcleo; icterus = icterícia) ${ }^{(31,44)}$.

Esses padrões, de um modo geral, se associam no mesmo paciente. Como exemplos, podem ser citados: hemorragias peri e intraventricular, e leucomalacia periventricular do prematuro. Como eles são mantidos vivos, às vezes, por conta de medidas terapêuticas extremas, as lesões cerebrais desses pacientes evoluem para cronicidade, com resolução da fase aguda. As alterações observadas à necropsia, como hidrocefalia pós-hemorragia e encefalopatia multicística não teriam oportunidade de se instalar sem o concurso desses vigorosos suportes terapêuticos.

É tradicional considerar o aparecimento de kernicterus (icterícia nuclear) apenas com níveis elevados de bilirrubinas (>20 mg/100 ml). No material de necropsia analisado, constatou-se que isso não ocorre em recém-nascidos prematuros, sobretudo quando estão infectados. Mesmo com níveis mais baixos, em torno de $10 \mathrm{mg} / 100 \mathrm{ml}$, eles apresentaram impregnação biliar dos núcleos cerebrais, apesar de se considerar que essa cifra está inserida numa faixa de segurança $a^{(16)}$.

Com esses dados, os neonatologistas ficam mais atentos no sentido de agir mais precocemente nesse grupo particular de pacientes, instituindo fototerapia e exsangüinotransfusão, evitando assim a encefalopatia pós-ictérica.

Não menos importante é a meningite neonatal, isto é, aquela que se instala até 28 dias após o nascimento, no período neonatal. Ela pode ser isolada ou, com grande freqüência, fazer parte de um quadro infeccioso sistêmico (Figura 3C).

Sob o ponto de vista etiológico, ela difere da meningite no lactente após três meses de vida. No recém-nascido, na maioria das vezes, é causada por enterobactérias gramnegativas, especialmente a Escherichia coli. Nas crianças maiores predomina o Haemophilus, Pneumococcus e Meningococcus. Isto se explica pelo fato de a fração 19-S da macroglobulina do soro, que contém anticorpos contra bactérias coliformes, não passar através da placenta, resultando na ausência de imunidade passiva adquirida para organismos gram-negativos no momento em que eles colonizam o trato gastrintestinal ${ }^{(17)}$.

Além disso, o padrão histopatológico é mais severo, notando-se leptomeningite e ventriculite em razão da maior exposição da fissura coróide no recém-nascido, condicionando, portanto, a possibilidade de comunicação do espaço subaracnóide com a cavidade ventricular ${ }^{(31)}$. A ventriculite dificulta o tratamento da infecção, sendo necessário administrar antibióticos por via intratecal.

A mortalidade nesses casos é muito alta e as seqüelas freqüentes. Associam-se fenômenos de flebotrombose com infarto, que se infectam posteriormente formando abscessos.

Foram estudados 16 casos de meningite neonatal - a maior parte por germes gram-negativos - adquiridas durante a internação, fazendo parte de quadros septicêmicos. O SNC foi comprometido em 12 dos 30 casos de sépsis fúngica (Figura $3 \mathrm{H}$ ).

No geral, as alterações nos vários sistemas são importantes, mas para os sobreviventes as lesões do SNC que definirão a qualidade de vida, no que se refere à aquisição das etapas motoras (sustentar cabeça, sentar, engatinhar e andar); as funções cognitivas e associativas; o bom funcionamento da visão e audição; o rendimento escolar, e o relacionamento na sociedade.

\section{Lesões cutâneas (Tabela 4)}

Devemos tecer ainda algumas considerações sobre lesões cutâneas que se distribuem num espectro que vai desde pequenas erosões circulares, com hiperemia produzida pelos eletrodos transcutâneos de oxigênio, até lesões lineares hiperêmicas resultantes de fitas adesivas usadas para fixação de prongas nasais, tubos nasotraqueais e/ou orotraqueais. Além de feridas puntiformes de venopunção 
em múltiplas localizações, feridas incisas para dissecação venosa, e até extensas lesões cutâneas resultantes de agentes químicos, como álcool iodado, produzindo lesões eritematobolhosas (Figura 2D).

Ao lado de lesões por agentes físicos e químicos, devem ser acrescentadas infecções cutâneas por bactérias gramnegativas, com formação de equimoses e flictenas; lesões impetiginosas por cocos piogênicos; e comprometimento cutâneo por Candida sp. Fenômenos trombembólicos levaram à necrose de região glútea (trombose de ilíaca primitiva), de pododáctilos e do pé.

\section{Nutrição parenteral (Tabela 8)}

Em 23 casos de nossa série havia lesão hepática relacionada com a nutrição parenteral. Um achado comum é a hiperbilirrubinemia conjugada e colestase, principalmente quando a alimentação parenteral se prolonga por mais de duas semanas. As crianças desenvolveram icterícia proporcional à gravidade da colestase, que inicialmente é centrolobular. Seguem-se o sofrimento hepatocitário e a expansão dos espaços porta, com fibrose, edema e proeminência das células de Kupffer. São observados também hepatócitos gigantes multinucleados com o progredir da lesão hepatocitária.

A modificação arquitetônica do fígado pode ser muito acentuada, havendo semelhança com os aspectos histológicos resultantes da atresia biliar extra-hepática.

Em recém-nascidos com colestase prolongada e fibrose hepática, pode haver depósito de ferro no fígado e em outros órgãos, como pâncreas, tireóide e hipófise, mimetizando assim os achados da hemossiderose idiopática neonatal.

No pâncreas, os ductos pancreáticos exibem secreção com intenso espessamento, com ocasional formação de microcálculos. Em casos mais graves, há evidências de fibrose difusa e atrofia do pâncreas exócrino. Mais uma vez, nessa alteração, o patologista deve ser cauteloso ao fazer o diagnóstico diferencial com a mucoviscidose.

A essa altura, fica clara a possibilidade de associação de múltiplas condições patológicas em um mesmo órgão e em múltiplos órgãos na mesma criança.
Assim, poderíamos ter um recém-nascido pré-termo, portador de doença de membrana hialina, submetido à ventilação assistida, que eventualmente desenvolve enfisema intersticial, com ou sem pneumotórax, e evolui para um quadro de displasia broncopulmonar ao longo de sua internação. Ao mesmo tempo, sua árvore respiratória alta pode ser lesada pelo manuseio e presença de tubos, facilitando uma infecção secundária, muitas vezes por germes de sua própria microbiota.

São comuns os episódios de hipotensão e falência circulatória, com a instituição de drogas vasopressoras. O quadro hipotensivo levaria a necroses miocárdica, renal e hepática, no SNC e/ou no trato gastrintestinal. Ao mesmo tempo, algum tipo de acesso vascular necessitará ser realizado a fim de se instituir drogas medicamentosas, fluidos e/ou nutrição parenteral total, com todos os possíveis efeitos adversos já anteriormente mencionados.

\section{Conclusão}

- A consulta e análise da literatura demonstram a virtual inexistência de metodologia adequada para desenvolver e estabelecer um protocolo capaz de oferecer um sistema de aferição do desempenho organizacional, que elimine todas as possibilidades de efeitos indesejáveis relacionados com as rotinas e procedimentos operacionais desse campo da prática assistencial.

- As principais causas desses problemas parecem ser creditadas à tecnologia de máquinas e substâncias, cuja adequação à biologia de organismos humanos em desenvolvimento não alcançou ainda níveis perfeitos de adaptação, no que concerne à influência exercida por um grande número de variáveis fisiopatológicas que atuam no procedimento terapêutico.

- A formulação de estratégias de melhoria da qualidade desse processo, tendo em vista as limitações impostas pelos princípios éticos da pesquisa que envolve os períodos pré e pós-natal, representa um desafio dentro das políticas de saúde que visam a universalização de procedimentos de alta complexidade.

- A necropsia, com adequada interpretação e correlação clínica das lesões, demonstrou ser um significativo instrumento de controle da qualidade das ações diagnósticas e terapêuticas executadas. 


\section{Referências}

1. BALAGTAS, R.C. et al. Risk of local and systemic infection associated with umbilical vein catheterization: a prospective study in 86 newborn patient. Pediatrics, v. 48, p. 359-67, 1971.

2. BEARDSALL, K. et al. Pericardial effusion and cardiac tamponade as complications of neonatal long lines: are they really a problem? Arch Dis Child Fetal Neonatal Ed (England), v. 88, n. 4, p. 292-5, 2003.

3. BIRKIN, B.B.; AMPLATZ, K. Jet collapse with aortic rupture: a complication of umbilical catheter angiography. Am J Roentgen Rad Ther Nucl Med, v. 116, p. 487-9, 1972.

4. BLEYER, W.A.; SKINNER, A.L. Fatal neonatal hemorrhage after maternal anticonvulsivant therapy. J AM Med Ass, v. 235, p. 626-7, 1976.

5. BOROS, S.J. et al. Necrotizing tracheobronchitis: a complication of high-frequency ventilation. J Pediatr, v. 109, p. 95-100, 1986.

6. BRADSHAW, W.T.; FURDON, S.A. A nurse's guide to early detection of umbilical venous catheter complications in infants. Adv Neonatal Care (United States), v. 6, n. 3, p. 127-38, 2006.

7. BRINSMEAD, M.W. Complications of amniocentesis. Med J Austral, v. 1, p. 379-85, 1976.

8. CHESSELS, J.M.; WIGGLESWORTH, J.S. Secondary hemorrhagic disease of the newborn. Arch Dis Child, v. 45, p. 539-43, 1970.

9. CORBET A. et al. Decreased mortality rate among small premature newborn infants treated with a single dose of synthetic surfactant: a multicenter controlled trial. J Pediatric, v. 118, p. 277-84, 1991.

10. CROSS, K.W. Cost of preventing retrolental fibroplasias. Lancet, v. 2, p. 954-6, 1973.

11. DE SA, D.J. The spectrum of ischemia bowel disease in the newborn. Perspect Pediat Pathol, v. 3, p. 273-309, 1976.

12. DE SA, D.J. Pathology of Neonatal Intensive Care. Chapman \& Hall Medical (USA), 1995, p. 54.

13. FAVARA, B.E.; MOORS, H.K. Foreing body embolism. Pediatric Pathol, v. 11, p. 371-80, 1991.

14. FOX, W.W. et al. Pulmonary hypertension in the perinatal aspiration syndromes. Pediatrics, v. 59, p. 205-10, 1977.

15. GAUGLER C. et al. Neonatal necrotizing tracheobronchitis: three case reports. J Perinatol (United States), v. 24, n. 4, p. 259-60, 2004.

16. GARTNER, L.H. et al. Kernicterus: high incidence in premature infants with low serum bilirrubin concentration. Pediatrics, v. 45, p. 906-17, 1970.

17. GITLIN, D.; ROSEN, F.S.; MICHAEL, J.G. Transient 19S gamma-1-globulin deficiency in the newborn infant, and its significance. Pediatrics, v. 31, p. 197-208, 1983.

18. GURWITZ, D. Chorionic villus sampling versus early amniocentesis. Lancet (England), v. 350, p. 1254-8.

19. GUTCHER, G.; CUTZ, E. Complications of parenteral nutrition. Sem Perinatol, v. 10, p. 196-207, 1986.

20. HERMANSEN, M.C.; HERMANSEN, M.G. Intravascular catheter complications in the neonatal intensive care unit. Clin Perinatol (United States), v. 32, n. 1, p. 141-56, 2005.

21. JEYABALAN, A.; CARITIS, S.N. Pharmacologic inhibition of preterm abor. Clin Obstet Gynecol, v. 45, p. 99-113, 2002.

22. KARP, L.E.; HAYDEN, P.W. Fetal puncture during midtrimester amniocentesis. Obst Gynaecol, v. 49, p. 115-7, 1977.

23. KULKARNI, P.B.; DORAND, R.D.; SIMMONDS JR., E.M. Pericardial tamponade: complications of total parenterall nutrition J Pediatr Surg, v. 16, p. 735-6, 1981.

24. LAMB, M.P. Gangrene of a fetal limb due to amniocentesis. Br J Obstet Gynaecol, v. 82,p. 829-30, 1975.

25. METLAY, L.A. et al. A new iatrogenic lesion in newborns requiring assisted ventilation. N Engl J Med, v. 309, p.111-2, 1983.

26. MIMONNE, F. et al. Necrotizing tracheobronchitis: case report. Pediatr, v. 77, p. 366-71, 1986.

27. MISENHIMER, H.R. Fetal hemorrhage associated with amniocentesis. Am J Obstet Gynecol, v. 94, p. 1133-5, 1966.

28. MOUTQUIN, J.M. Treatment of preterm labour with the beta-adrenergic agonist ritodrine. N Englan J Med, v. 327, p. 308-12, 1992.

29. MULLICK, F.G.; MORAN, C. Adverse drug reactions in pediatric pathology. Philadelphia: Lippincott, 1992; p. 239-55.

30. MURPHY, J.D. et al. Structural basis of persistent pulmonary hypertension of the newborn infant. J Pediar, v. 98, p. 962-70, 1981.

31. NORONHA, L. et al. A neuropatologia no período neonatal. Arq Neuropsiquiatria, v. 59, p. 411-6, 2001.

32. PEDEN, V.H.; WITZLEBEN, C.L.; SKELTON, M.A. Total parenteral nutrition. J Pediatr, v. 78, p. 180-1, 1971.

33. REDLINE, R.W.; DAHMS, B.B. Malassezic pulmonary vasculitis in an infant on long-term intralipid therapy. N Engl J Med, v. 305, p. 1395-6, 1981.

34. REDLINE, R.W. et al. Systemic Malassezia furfur infections in patients receiving intralip therapy. Hum Pathol, v. 16, p. 815-23, 1985.

35. REHDER, H. Fetal limb deformities due to amniotic constrictions: a possible consequence of proceding amniocentesis. Pathol Res Pract, v. 162, p. 316-26, 1978.

36. ROGERS, B.B. et al. Pericardial tamponade secondary to central venous catheterization and hyperalimentation in a very low birth weight infant. Pediatric Pathol, v. 10, p. 819-33, 1990.

37. RYAN, G.T. et al. Fetal bleeding as a major hazard of amniocentesis. Obstet Gynecol, v. 40, p. 702-7, 1972.

38. SERAPIÃO, M. Curso prático de necropsia perinatal. Curitiba: Anais do XXII Congresso Brasileiro de Patologia, 1999.

39. SERAPIÃO, M.; SERAPIÃO, M.J.; SERAPIÃO, C.J. Tamponamento pericárdico secundário à cateterização venosa central para alimentação parenteral com 
Intralipid em recém-nascidos de baixo peso: estudo de dois casos. Buenos Aires: Anais da IX Conferencia Latinoamericana de Patologia Pediátrica, 2000.

40. SERAPIÃO, M.; SERAPIÃO, M.J.; SERAPIÃO, C.J. Complicações do cateterismo arterial e venoso em UTI neonatal. Buenos Aires: Anais da IX Conferencia Latinoamericana de Patologia Pediátrica, 2000.

41. THERKENSEN, A.J.; REHDER, H. Intestinal atresia caused by second trimester amniocentesis. Br J Obster Gynaecol, v. 88, p. 559-62, 1981.

42. VALDES-DAPENA, M. latrogenic disease in the perinatal period as seen by the pathologist in Perinatal Disease. Baltimore: Williams and Wilkins. 1981; p. 382.
43. WHO/PAHO CONSULTATION ON CVS. Evaluation of chorionic villus sampling safety. Prenat Diagn (England), v. 19, p. 97-9, 1999.

44. WIGGLESWORTH, J.S. Perinatal pathology. Philadelphia: WB Saunders. 1984; p. 230-1.

45. WIGGLESWORTH, J.S.; SINGER, D.B. Fetal and perinatal brain damage in Text Book of Fetal and Perinatal Pathology. Oxford: Blakwell Scientific Publications. 1988; p. 80738.

46. YOUROUKOS, S. et al. Porencephalic cysts after amniocentesis. Arch Dis Child, v. 55, p. 814-5, 1980.

\begin{tabular}{l|l} 
& Endereço para correspondência \\
\hline & Mônica Serapião \\
& Serviço de Patologia do Hospital Dona Helena \\
& Rua Blumenau, 123 - Centro \\
CEP 89204-250 - Joinville-SC \\
e-mail: serapiao@terra.com.br
\end{tabular}

